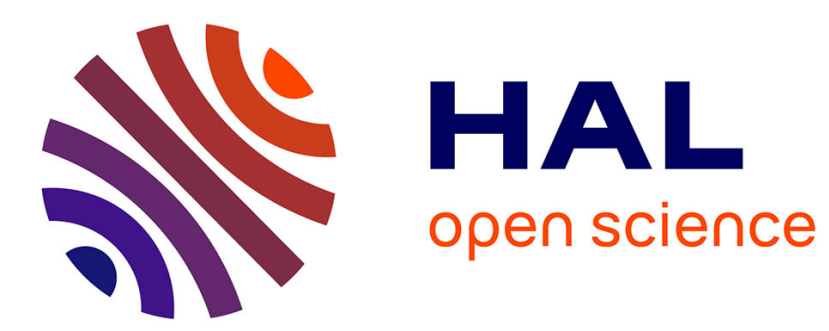

\title{
MODÉLISATION DE MOTEURS PIÉZOÉLECTRIQUES MODULAIRES
}

\author{
Jean Dion, M. Brissaud
}

\section{To cite this version:}

Jean Dion, M. Brissaud. MODÉLISATION DE MOTEURS PIÉZOÉLECTRIQUES MODULAIRES. Journal de Physique IV Proceedings, 1992, 02 (C1), pp.C1-373-C1-376. 10.1051/jp4:1992180 . jpa00251252

\section{HAL Id: jpa-00251252 https://hal.science/jpa-00251252}

Submitted on 1 Jan 1992

HAL is a multi-disciplinary open access archive for the deposit and dissemination of scientific research documents, whether they are published or not. The documents may come from teaching and research institutions in France or abroad, or from public or private research centers.
L'archive ouverte pluridisciplinaire HAL, est destinée au dépôt et à la diffusion de documents scientifiques de niveau recherche, publiés ou non, émanant des établissements d'enseignement et de recherche français ou étrangers, des laboratoires publics ou privés. 


\title{
MODÉLISATION DE MOTEURS PIÉZZÉLECTRIQUES MODULATRES
}

\author{
J.L. DION et M. BRISSAUD* \\ Laboratoire d'Ultrasonique, Université du Québec à Trois-Rivières, CP. 500, Trois-Rivières, Québec \\ G9A 5H7, Canada \\ *Laboratoire de Génie Electrique et Ferroélectricité, Bâtiment 504, Institut National des Sciences \\ Appliquées de Lyon, 20 avenue Albert Einstein, F-69621 Villeurbanne, France
}

\begin{abstract}
A one-dimensional analysis is presented of piezoelectric motor stator modules composed of elements vibrating simultaneously in longitudinal and transverse modes. A rigourous transmission line analogy is used throughout to determine coincidence of both resonance modes, considering or not the effect of cement layers. It is shown that this type of structure should be applicable if this last effect could be eliminated. Good agreement with experimental values is found for simple structures.
\end{abstract}

\section{INTRODUCTION}

Depuis quelques années, on a fait de nombreuses études sur divers types de moteurs piézoélectriques qui suscitent de l'intérêt [1-4]. En principe, de tels moteurs peuvent produire des forces importantes à basse vitesse d'une façon contrôlée, ce qui les rend intéressants par rapport aux moteurs électromagnétiques avec réducteurs de vitesse. On peut appeler moteur piézoélectrique modulaire une structure formée par diverses associations de céramiques piézoélectriques (fig. 1) vibrant en épaisseur (1,2) et d'autres vibrant en cisaillement (3-4), avec des couches solides accolées (5-6-9). Les deux types de céramiques sont excités par des sources de tension $V_{1}$ et $V_{2}$ mutuellement déphasées. Une telle structure constitue un résonateur composé pouvant résonner simultanément suivant deux axes perpendiculaires $Y-Z$ et produire ainsi un mouvement superficiel elliptique qui cause le déplacement d'une pièce mobile (7) en contact Nous n'avons relevé qu'une seule structure apparentée dans la bibliographie [5]. Mais celle-ci doit présenter en pratique des problèmes de raccordement électrique. Pour l'analyse, nous avons supposé que les vibrations se font en une dimension et utilisé une méthode découlant de l'analogie avec les lignes électriques et d'un modèle particulier de transducteur piézoélectrique [6]. Nous avons pu vérifier expérimentalement au voisinage de $100 \mathrm{kHz}$ que cette modélisation est très satisfaisante dans la mesure où elle permet de prédire les fréquences de résonance avec une précision de l'ordre de $1 \%$ et les impédances électriques à mieux de $5 \%$ pour des structures de quatre ou cinq couches. Elle nous a aussi permis de prédire l'effet radical des couches de colle observé en pratique. Il s'est avéré que même en couches inférieures à $50 \mu \mathrm{m}$, les colles cyanoacrylates avaient un effet amortisseur excessif pour cette application. Nous croyons que ce problème peut être réglé au moyen de colles à grandes performances, utilisables en couches inférieures à $10 \mathrm{~mm}$, ou par une technique de brazage en couche mince. Nous présentons un choix de simulations typiques démontrant les possibilités éventuelles de ce type de moteur piézoélectrique: calculs d'impédance électrique, de vitesse acoustique.

\section{MÉTHODE D'ANALYSE}

Idéalement, le système de la figure 1 peut se représenter comme dans la figure 2 , si l'on néglige les couches de colle, ou comme dans la figure 3 avec les couches de colle, supposant que la propagation d'énergie se fait en une dimension seulement suivant l'axe Y. De cette façon, nous pouvons utiliser l'analogie avec la propagation des ondes de courant et de tension sur les lignes électriques et utiliser l'ensemble des relations mathématiques qui s'y appliquent. La figure 3 montre le sytème comme une chaine de lignes acoustiques. Les effets des éléments piézoélectriques 9, 10,12 et 13 sont alors superposés. Dans l'étape illustrée, l'élément 10 est actif. La représentation utilisée pour cet élément a été introduite dans des publications précédentes [6,7]. On y démontre que la relation entre la tension appliquée $V$, le courant $I$ et les vitesses acoustiques $U$ des faces A,B de l'élément 10 est: 


$$
V=-\frac{h}{j \omega}\left(U_{\mathrm{B}}-U_{\mathrm{A}}\right)+\frac{1}{j \omega C_{\mathrm{o}}} I
$$

où $h$ est la constante piézoélectrique, $\omega$ la pulsation et $C_{\mathrm{o}}$ la capacité bloquée. L'impédance acoustique vue vers la droite (sens + ) est de la forme:

$$
Z_{+}=\frac{Z_{\mathrm{d}} \cosh (\gamma a)+Z_{\mathrm{o}} \sinh (\gamma a)}{Z_{\mathrm{o}} \cosh (\gamma a)+Z_{\mathrm{d}} \sinh (\gamma a)} Z_{\mathrm{o}}
$$

où $Z_{\mathrm{d}}$ est l'impédance acoustique vue à droite de l'élément suivant d'épaisseur $a$ et d'impédance caractéristique $Z_{0}, \gamma$ étant la fonction de propagation: $\gamma=\alpha+\mathrm{j} \beta, \alpha$ est le coefficient d'atténuation du milieu et $\beta=\omega / v$, avec $v$ la vitesse de phase. On a une expression semblable $Z$. pour l'impédance vue vers la gauche de l'élément 10. La gauche et la droite sont définis par rapport au sens de propagation de l'énergie acoustique à partir de l'élément actif. D'autre part, la relation entre la vitesse $U_{\mathrm{g}}$ à gauche d'une ligne et la vitesse $U_{\mathrm{d}}$ à droite est la suivante:

$$
U_{\mathrm{g}}=\left[\cosh (\gamma a)+\left(Z_{\mathrm{d}} / Z_{\mathrm{o}}\right) \sinh (\gamma a)\right] U_{\mathrm{d}}
$$

On obtient également des formes explicites de l'impédance électrique présentée par un élément piézoélectrique, ainsi que des vitesses acoustiques de ses faces gauche (A) et droite (B):

$$
\begin{array}{ll}
Z_{\mathrm{E}}=\frac{1}{j \omega}\left(R_{\mathrm{B}}-R_{\mathrm{A}}\right)+\frac{1}{\mathrm{j} \omega C_{\mathrm{o}}} \\
U_{\mathrm{A}}=\frac{R_{\mathrm{A}}}{Z_{\mathrm{E}}} V \quad U_{\mathrm{B}}=\frac{R_{\mathrm{B}}}{Z_{\mathrm{E}}} V
\end{array}
$$

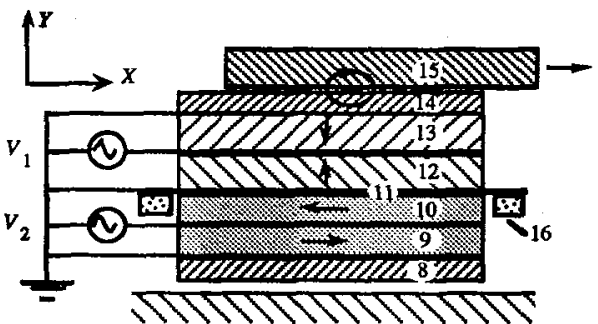

Figure 1 Moteur piézólectrique modulaire

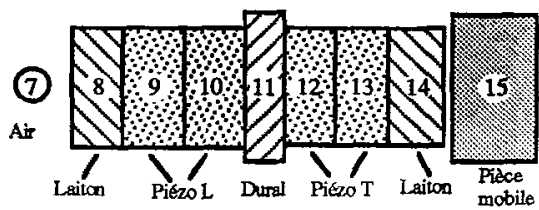

Figure 2 Struchure du stator sans colle

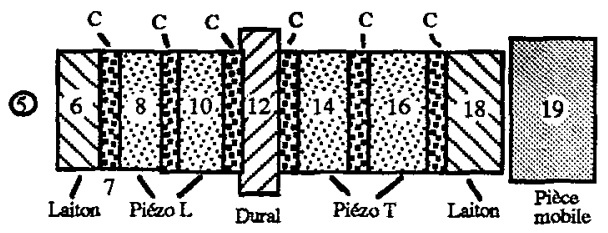

Figure 3 Structure du stator avec colle

où $R_{\mathrm{A}}$ et $R_{\mathrm{B}}$ sont des fonctions faisant intervenir les divers paramètres piézoélectriques et acoustiques du système $[6,7]$.

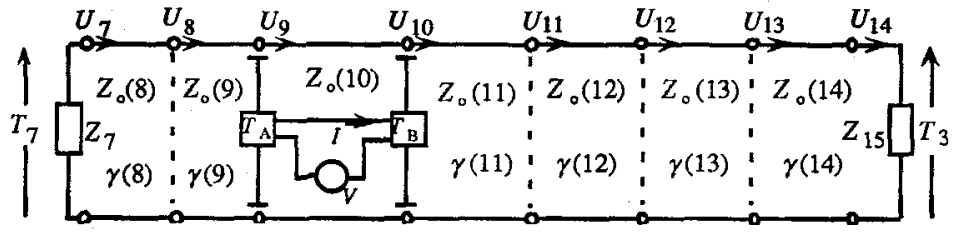

Figure 4

Modèle en forme de ligne du système des figures 1 ou 2 . L'élément piézoélectrique actif porte ici le numéro 10.

\section{RÉSULTATS}

Nous avons élaboré des logiciels permettant de calculer les spectres de vibration dans les deux modes (longitudinal et transversal) pour une vingtaine de couches, y compris les couches de liaison (ciment). Ils fournissent l'impédance électrique du moteur et les vitesses acoustiques des diverses interfaces. Cette modélisation monodimensionnelle permet de prévoir par calcul la combinaison d'épaisseurs telle que les résonances longitudinales et transversales coïncident, réalisant le rendement maximal du système. Les résonances en d'autres dimensions produisent en pratique des perturbations qui affectent relativement peu la position et l'amplitude des résonances prévues par le modèle en une dimension. La figure 5 montre un premier système plus simple à cinq couches qui fut calculé puis réalisé. Les deux piézocéramiques 10 et 14

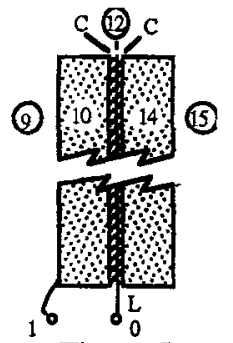

Figure 5

vibrant en épaisseur sont décrites dans le tableau 1, de même que les couches de colle $C$. Le feuillard de laiton $L$ a une épaisseur de $25 \mu \mathrm{m}$. Les figures 6-a et 6-b montrent un bonne correspondance entre la théorie et l'expérience pour l'impédance électrique 1-0, à l'appui du modèle. 
TABLEAU 1 - Propriétés acoustiques et mécaniques

\begin{tabular}{|c|c|c|c|c|}
\hline & Piézocéram. P-762 * & Duralumin & Laiton & Colle ${ }^{* *}$ \\
\hline Epaisseur (mm) & Voir tableau 2 & 1,5 & 3 & 0,040 \\
\hline Surface $\quad\left(\mathrm{cm}^{2}\right)$ & 2,592 & 2,592 & 2,592 & 2,592 \\
\hline Masse vol. $\left(\mathrm{kg} / \mathrm{m}^{3}\right)$ & 7517 & 2790 & 8600 & 1140 \\
\hline$v$ longit. II $(\mathrm{m} / \mathrm{s})$ & 4490 & 6300 & 4700 & 2100 \\
\hline$v$ longit. $\dagger(\mathrm{m} / \mathrm{s})$ & 4140 & 6320 & 4700 & 2100 \\
\hline $\begin{array}{l}v \text { transv. } \|(\mathrm{m} / \mathrm{s}) \\
v \text { transv. }+(\mathrm{m} / \mathrm{s})\end{array}$ & $\begin{array}{l}1843 \\
2355\end{array}$ & 3130 & 2110 & 500 \\
\hline$Z_{\mathrm{oL}} \quad$ (rayls) & $33,75 \cdot 10^{6}$ & $17,63 \cdot 10^{6}$ & $40,42 \cdot 10^{6}$ & $2,40 \cdot 10^{6}$ \\
\hline (rayls) & $17,55 \cdot 10^{6}$ & $8,733 \cdot 10^{6}$ & $18,15 \cdot 10^{6}$ & $5,7 \cdot 10^{5}$ \\
\hline$c_{33}^{\mathrm{D}} \quad \mathrm{N} / \mathrm{m}^{2}$ & $1,515 \times 10^{11}$ & $1,114 \times 10^{11}$ & $1,900 \times 10^{11}$ & $0,050 \times 10^{11}$ \\
\hline$c_{44}^{\mathrm{D}} \quad \mathrm{N} / \mathrm{m}^{2}$ & $4,132 \times 10^{10}$ & $2,733 \times 10^{10}$ & $3,829 \times 10^{10}$ & $0,092 \times 10^{10}$ \\
\hline $\begin{array}{l}\alpha \text { longit. }(\mathrm{Np} / \mathrm{m}) \\
\text { à } f_{\text {ref }}=527 \mathrm{kHz}\end{array}$ & 8 & 3 & 10 & 75 \\
\hline $\begin{array}{l}\alpha \text { transv. }(\mathrm{Np} / \mathrm{m}) \\
\text { a } f_{\text {reff }}=583 \mathrm{kHz}\end{array}$ & 10 & 8 & 16 & 90 \\
\hline * Quartz et Silic & ** Loctite 307 & & & \\
\hline
\end{tabular}

TABLEAU 2 - Propriétés électriques et piézoélectriques Pí́zocéramique P-762

\begin{tabular}{|c|l|}
\hline Vibration longitudinale & \multicolumn{1}{|c|}{$\begin{array}{c}\text { Vibration transversale } \\
\text { Polarisation perpendiculaire aux électrodes }\end{array}$} \\
\hline Épaisseur $a=4,265 \mathrm{~mm}$ & Epaisseur $a=2,02 \mathrm{~mm}$ \\
$C_{\mathrm{o}}=380 \mathrm{pF} \quad h_{33}=2,564 \cdot 10^{9} \mathrm{~N} / \mathrm{C}$ & $C_{\mathrm{o}}=908 \mathrm{pF} \quad h_{15}=1,457 \cdot 10^{9} \mathrm{~N} / \mathrm{C}$ \\
$D=0,0073$ (facteur de pertes) à $1100 \mathrm{kHz}$ & $D=0,017 \mathrm{a} 1200 \mathrm{kHz}$ \\
$\varepsilon_{33}^{\mathrm{S}}=6,265 \times 10^{-9} \mathrm{~F} / \mathrm{m} \quad k_{33}^{\mathrm{t}}=0,521$ & $\varepsilon_{11}^{\mathrm{S}}=7,030 \times 10^{-9} \mathrm{~F} / \mathrm{m}$ \\
& $k_{15}=0,601$ \\
\hline
\end{tabular}

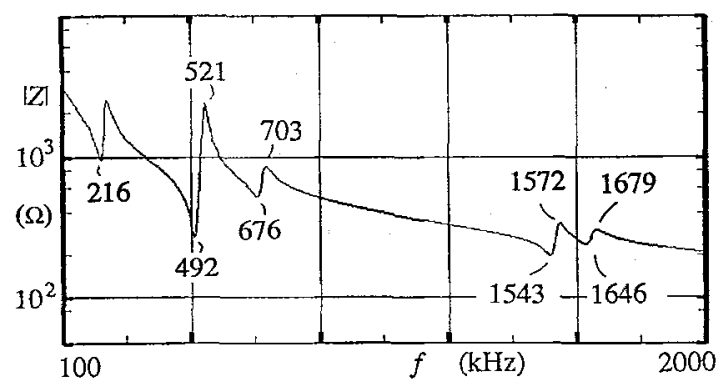

Figure 6-a

Impédance électrique calculée de l'élément 10 du module de la figure 5 .

Figure 6-b

Impédance électrique mesurée de l'élément 10 du module de la figure 5 . Les résonances superflues sont causées par les modes latéraux.

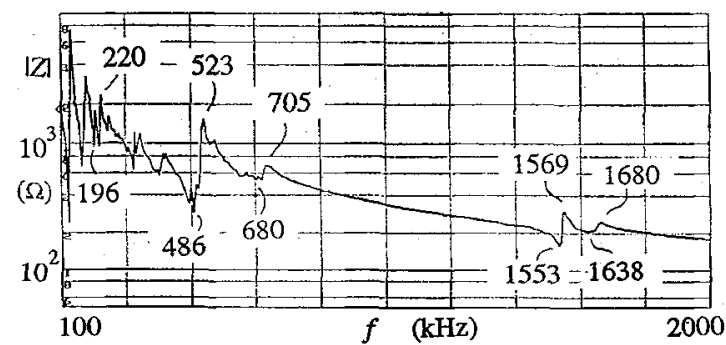


On peut voir à la figure 7 les vitesses acoustiques calculées, longitudinale et transversale, de la face 14 du système de la figure 2 (sans colle) quand la tension appliquée est de 1 volt. On a supposé que la pièce mobile (milieu 15) présentait une impédance équivalente de 2 Mrayls. Les épaisseurs des couches de laiton et de duralumin ont été choisies de façon à faire coïncider les résonances longituđinale et transversale à 97 $\mathrm{kHz}$ (voir le tableau 1). Comme le modèle tient compte des pertes dans les divers milieux, on peut évaluer un rendement d'environ $80 \%$ dans ce cas. Les divers paramètres acoustiques de la colle ayant été déduits des mesures et calculs se rapportant au système de la figure 5 , on a ensuite calculé la structure de la figure 3. On peut alors prédire un effet assez radical des couches de colle de $40 \mu \mathrm{m}$, comme l'indique la figure 8 , particulièrement pour la résonance transversale: celle-ci tombe à $62,2 \mathrm{kHz}$ (autre mode: $84,4 \mathrm{kHz}$ ).

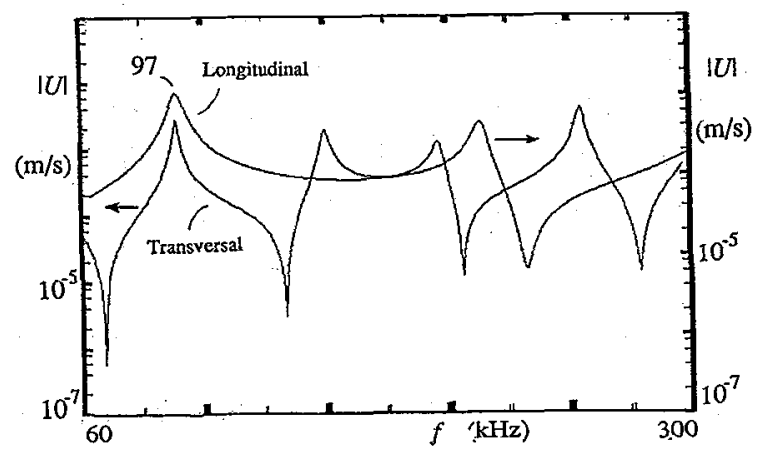

Figure 7

Spectres de vitesse acoustique calculés de la structure des figures 1 ou 2 (sans colle).

Les deux modes de résonance coïncident à 97 $\mathrm{kHz}$. La résonance longitudinale est la première. La résonance transversale est la deuxième: la première est à moins de $60 \mathrm{kHz}$.

Figure 8

Spectres de vitesse acoustique calculés de la $(\mathrm{m} / \mathrm{s})$ structure des figures 1 ou 2 (avec colle).

La résonance longitudinale passe de 97 à 84,4 kHz. La résonance transversale passe de 97 a $62,2 \mathrm{kHz}$.

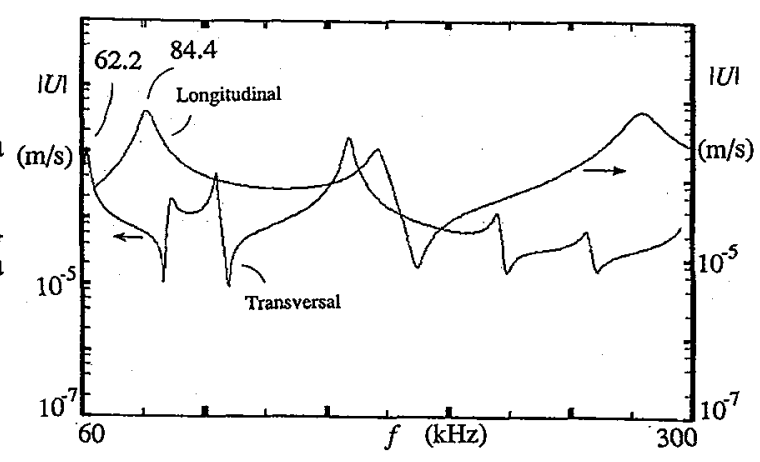

CONCLUSION Ce modèle de moteur piézoélectrique a permis d'expliquer, en tenant compte des couches de colle, pourquoi les quelques structures réalisées par collage ne fonctionnaient pas comme prévu. Les couches de colle, même en couches inférieures à $50 \mu \mathrm{m}$, ont un effet amortisseur extrême, particulièrement en cisaillement, comme on pouvait s'y attendre. Les calculs indiquent que leur remplacement par de la soudure en couche mince pourrrait régler le problème.

\section{BIBLIOGRAPHIE}

[1] T. KAMANO, T. SUZUKI et E. OTOI, Characteristics and model of ultrasonic motor, Proc. 8th Symp. on Ultrasonics Electronics, Tokyo 1987, Jap. J. Appl. Phys., v. 27 (1988), suppl. 27-1, 189-191.

[2] M. FLEISCHER, D. STEIN, H. MEIXNER, New type of piezoelectric motor, IEEE Trans. Ultrasonics, Ferroelectrics, Freq. Control, vol. 36, No. 6 (1989), 614-619.

[3] T. UCHIKAWA, O. MYOHGA, O. OHNISHI, M. TAMEGAI, T. INOUE et S. TAKAHASHI, Small-diameter hightorque ultrasonic motor using longitudinal-torsional composite vibration, Ultrasonics internat. 1989, Madrid.

[4] O. KAWASAKI, K. TAKEDA et T. NISHIKURA, Ultrasonic Motor, Office Européen des Brevets, № 0315933 A2, 17 mai 1989, Bulletin 89/20.

[5] K. SUZUKI, Piezoelectric drive device, No de dépót 58-164333, Office Japonais des Brevets, 6 avril 1985.

[6] DION, J.L., Un nouveau modèle unidimensionnel de transducteur piézoélectrique, Premier Congrès Français d'Acoustique, Lyon, avril 1990.

[7] J.L. DION, Jean-Luc, A new approach to one-dimensional piezoelectric transducer modelling, IEEE-90 Ultrasonics Symposium, Honolulu, Hawai, décembre 1990. 